\title{
Estimating the Marginal Causal Effect of Fish Consumption during Adolescence on Multiple Sclerosis: A Population-Based Incident Case-Control Study
}

\author{
Ibrahim Abdollahpour ${ }^{\mathrm{a}}$ Saharnaz Nedjat ${ }^{\mathrm{b}}$ Mohammad Ali Mansournia ${ }^{\mathrm{c}}$ \\ Mohammad Ali Sahraian ${ }^{d}$ Jay S. Kaufman ${ }^{\mathrm{e}}$ \\ ${ }^{a}$ Department of Epidemiology, School of Public Health, Arak University of Medical Sciences, Arak, Iran; ${ }^{b}$ Department \\ of Epidemiology and Biostatistics, School of Public Health, Tehran University of Medical Sciences, Knowledge \\ Utilization Research Center, Tehran University of Medical Science, Tehran, Iran; ' Department of Epidemiology

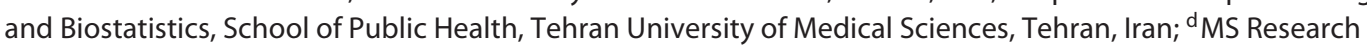

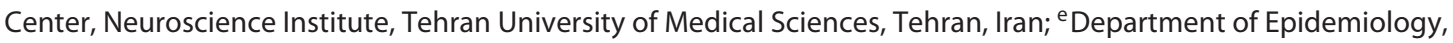 \\ Biostatistics, and Occupational Health, McGill University, Montreal, QC, Canada
}

\author{
Keywords \\ Causal analysis - Case-control study · Inverse-probability- \\ of-treatment weighting · Model-based standardization . \\ Multiple sclerosis · Fish intake
}

\begin{abstract}
Background: Adolescence is considered as a critical time period in multiple sclerosis (MS) etiology. Nonetheless, there are insufficient reports regarding the potential role of fresh and canned fish consumptions during adolescence in MS etiology. The authors investigated the association between fresh and canned fish consumptions and MS. Methods: This was a population-based incident case-control study conducted in Tehran. Cases $(n=547)$ identified from Iranian Multiple Sclerosis Society between August 7, 2013, and November 17, 2015 were included in the study. Population-based controls $(n=1,057)$ were recruited by random digit telephone dialing without any matching. Inverse-probability-of-treatment weighing (IPTW) using 2 sets of propensity scores and model-
\end{abstract}

\section{KARGER}

(c) 2018 S. Karger AG, Basel

E-Mail karger@karger.com

www.karger.com/ned based standardization were used to separately estimate the marginal odds ratio between fresh and canned fish consumptions in adolescence and MS. Results: The marginal OR for fresh fish was $0.72(95 \% \mathrm{Cl} 0.58-0.90 ; p=0.005)$ in both IPTW analyses. Similarly, the marginal OR for canned fish consumption was $0.75(95 \% \mathrm{Cl} 0.60-0.95 ; p=0.014)$. The model-based standardized OR was $0.72(95 \% \mathrm{Cl} 0.58-0.91 ; p=0.008)$ for fresh and $0.73(95 \% \mathrm{Cl} 0.59-0.94 ; p=0.006)$ for canned fish consumption in adolescence. Discussion: Subject to limitation of case-control studies in interpreting associations causally, this study suggests that both fresh and canned fish consumptions in adolescence can decrease the risk of MS.

(c) 2018 S. Karger AG, Basel

\section{Introduction}

The incidence and prevalence of multiple sclerosis (MS) as a leading cause of non-traumatic disability in young adults has increased recently [1]. A similar pattern 
in the age-adjusted incidence and prevalence of MS has been reported in various parts of Iran $[2,3]$.

There are consistent reports demonstrating the potential role of some environmental factors including passive, tobacco, and waterpipe smoking [4-6] as well as vitamin $\mathrm{D}[7]$ in the poorly understood etiology of MS. However, the potential role of nutrition components as modifiable lifestyle factors, including fish intake, meat, fruit and vegetable consumption, in MS etiology is still not wellacknowledged. Similarly, the marginal causal effect of fresh and canned fish consumptions in adolescence, as a critical lifetime period for MS development, has not been studied [8].

There are several causal methods available for the analysis of case-control studies [9-13]. For example, inverseprobability-of-treatment weighting (IPTW) using propensity score (PS) [12] and model-based standardization (MBS) with the total population as the standard $[9,10,14]$ have been previously used for estimating marginal causal effects in case-control studies. The aim of this study was to estimate the marginal causal effect of fresh and canned fish consumptions in adolescence on MS using IPTW and MBS in the setting of a population-based incident casecontrol study with known sampling fractions (SF) for both case and control groups.

\section{Methods}

This was a population-based incident case-control study of all residents of 22 municipal areas of Tehran aged 15-50 years between August 7, 2013 and November 17, 2015 (nearly 5.11 million persons) as the study primary base [4]. We used the Iranian Multiple Sclerosis Society (IMSS), as the only registry in Tehran for recruitment of incident cases. For estimating the completeness of case recruitment via IMSS, the expected number of incident cases during the study period was calculated. Based on the latest report in Tehran in 2013, the age-adjusted incidence rate was 6.6 per 100,000 per year [15]. Then, with a population of 5.11 million, the occurrence of 506 new cases (337 new cases per year $\times 1.5$ years) is expected. Confirmed diagnosis of MS was established by at least one neurologist using $2010 \mathrm{McD}$ onald criteria [16] as well as the use of MRI. Index date was defined as the year and month in which the patients received her/his confirmed diagnosis. The sampling fraction for cases was 0.96. Using random digit dialing (RDD), 1,057 general population controls aged 15-50 years who were, at the time of case diagnosis, alive and in the study base participated in the study. We selected controls proportional to the size for 22 areas of Tehran. In the case of nonresponse, another control was selected using the same protocol. The sampling fraction for controls was $(1,057 / 5,115,679)=$ 0.00020661968 . The study was approved by the "ethics in research committee" of Tehran University of Medical Science with approval number 26145 .

\section{RDD Protocol}

We used the standard method of RDD for identifying eligible controls [17]. For this, the 22 existing area codes were completed by four randomly generated digits. If the completed number was an active residential phone, the study interview was started; else, the number was discarded. The similarity of selected controls using a RDD approach with selected controls using addressbased sampling has been previously shown [18]. Moreover, its usefulness, effectiveness, and feasibility have also been previously demonstrated $[17,19]$. Based on our RDD protocol, to identify and select potential controls, a maximum of 9 calls ( 2 times in am, 2 times in pm and 5 times at different times of the other days) were made before a randomly generated phone number could be discarded. We excluded non-residential numbers and finally reached a total of 1,591 residential numbers during our RDD process (approximately $36.6 \%$ of the generated random digits gave residences). A screening interview, to determine whether any household member met the study criteria, was conducted before the main study interview during the same call. We applied the Kish method for random selection of eligible members of each selected household [20, 21].

\section{Data Collection}

Dietary information was extracted using the food-frequency questionnaire as a part of a questionnaire for environmental risk factors in multiple sclerosis [22]. We obtained the average frequency of fresh and canned tuna fish intake during adolescence as well as the average amount consumed (usual portion size) in each meal. We exchanged the extracted information to the relevant standard serving size. We defined a half of a $200 \mathrm{~g}$ canned tuna fish and one slice of fish (100-120 g) as one standard serving size [23]. The phone interviews were conducted by 10 interviewers, selected for their strong communication and interview skills, and trained to use the standardized data collection procedures. We systematically tried to extract data from case and control groups by avoiding any interviewer bias and standardizing the data collection process.

\section{Exposure}

Data on the average frequency and average amount of fresh and canned tuna fish consumptions over adolescence were separately extracted by asking how many times per week, on average, they consumed a particular food when they were 13-19 year old. For the specific amount consumed, we asked for the typical serving size at each meal when they were 13-19 years old. Although detailed data on the other food items were also obtained, only the marginal causal effects of fresh and canned tuna fish consumptions were investigated in this study.

\section{Confounders}

Based on the causal diagram [24] in Figure 1, the following covariates were selected as confounders for the effect of fresh and canned fish consumptions over the period of adolescence on MS and adjusted for in IPTW and MBS: gender, age, socioeconomic status (SES), sunlight exposure in adolescence, physical activity in adolescence, smoking in adolescence, and passive smoking in adolescence.

We measured physical activity in adolescence using the International Physical Activity Questionnaire [25] along with a questionnaire for environmental risk factors in multiple sclerosis [22]. We obtained intensity level (vigorous or moderate), frequency per 


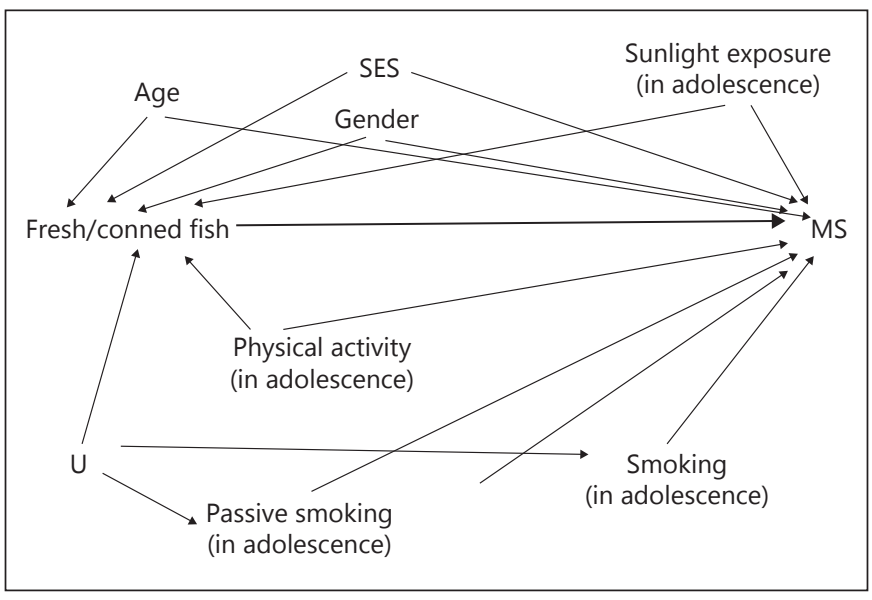

Fig. 1. A causal diagram representing the effect of fresh and canned fish consumptions on MS in the source population. To avoid clutter and without loss of validity of backdoor criterion, the arrows between confounders were not shown.

week, and average duration ( $\mathrm{min}$ ) of participants' activities during their adolescence. The metabolic equivalent of task (MET) was calculated by multiplying frequency and duration values for vigorous and moderate physical activities times MET intensity ( 8 for severe and 4 for moderate activities), respectively. The total MET-min/ week was estimated by the sum of calculated METs across 2 stated activity types [26]. Then, the total METs used in the data analysis were calculated for moderate-to-vigorous activities. The total MET-min/week was recoded to a binary variable (acceptable $[\geq 2,520 \mathrm{MET}$-min/week] and not acceptable $[<2,520 \mathrm{MET}-\mathrm{min} /$ week]) as recommended by Strong et al. [27].

Sunlight exposure in adolescence (age 13-19 years) [22] was separately extracted asking the following question: In adolescence (age 13-19 years), how many minutes/day in average did your activities (playing, participating in sports, watching sports, shoveling snow, walking, work activities, etc.) take you outside? Information on smoking behavior in adolescence, as a well-known risk factor for MS, was collected asking the following question: Have you smoked regularly when you were 13-19 years old? We also obtained information related to passive smoking (ever lived with anyone who regularly smoked) in individuals' adolescence.

SES was measured using a 10 stairs ladder as a subjective visual scale [28]. The data were extracted using the following instruction: "Imagine a ladder with 10 stairs representing where people stand in Tehran when you were adolescent (13-19 years old). At the top level of the ladder, there are those with the most money and the highest education and job situation. Conversely, at the bottom, there are those with the least money, the poorest education, and the worst job situations, that is, the higher the stairs, the better the social status and vice versa". The participants were then requested to select the stair that best shows their socioeconomic status in Tehran society at that time [28]. There were a self-reported questions asking the family history of MS, as well as 16 autoimmune diseases that is, infectious mononucleosis, migraine, lupus, hypothyroid, hyperthyroid, rheumatoid arthritis, Crohn's disease, colitis, type 1 diabetes, celiac, leukemia, Hodgkin's lymphoma, nonHodgkin's lymphoma, melanoma, non-melanoma skin cancer, and kidney disease. The participants were requested to give all aforementioned information before the index date for cases and the sampling date for controls.

\section{Statistical Methods}

Two different methods were used for estimating marginal causal ORs: IPTW and MBS.

\section{Inverse-Probability-of-Treatment Weighting}

Two sets of PS (i.e., the probability of fresh fish consumption in adolescence conditional on covariates) were estimated by fitting 2 logistic regression models with fresh fish consumption in adolescence as response and confounders as predictors among (i) controls, and (ii) the total sample with inverse-probability weighting where weights are the inverse of case and control SF, that is, $(1 / 0.96)=1.042$ for cases and $1 /(1,057 / 5,115,679)=4,839.81$ for controls $[29,30]$. Then the marginal causal effect of fresh fish consumption in adolescence was estimated using an inverse-probability-of-treatment weighted logistic regression analysis with case/ control as response and fresh fish consumption in adolescence as the only predictor with weights equal to (marginal prevalence of exposure)/PS for exposed and (1-marginal prevalence of no exposure) $/(1-P S)$ for unexposed. The same steps were then repeated for canned fish consumption as the second main exposure in this study. A robust 'sandwich' standard error was used to derive 95\% CI.

The consistency of the inverse-probability-of-treatment weighted estimates using method (i) and (ii) rely on the assumptions of no unmeasured confounding, positivity, and correct exposure model specification. Method (i) further requires the rare disease assumption which holds for MS since its prevalence in Tehran ranged from 5.3 to 74.28 per 100,000 [31]. We excluded covariates associated with fresh or canned fish consumption in adolescence but not with MS from the exposure model in the interests of efficiency and positivity [32]. Also we included variables which are not related to the fresh or canned fish consumption in adolescence but strongly associated with the MS (e.g., passive smoking), because this increases the precision of the effect estimate without increasing bias [33]. We used background knowledge for verifying whether the covariates are risk factors for MS. The associations between the covariates and fresh and canned fish consumptions in adolescence were evaluated in the study controls [9].

\section{Model-Based Standardization}

To estimate the marginal causal effect of fresh and canned fish consumptions during adolescence on MS, we also used a MBS method (sometimes known as the parametric g-formula) with the total source population as the standard $[9,34]$. First, we fitted the outcome logistic regression model with case/control status as the response and fresh fish consumption in adolescence and confounders as predictors and corrected intercept as "apparent intercept - case sampling fraction/control sampling fraction" where case sampling fraction/control sampling fraction $=\log (0.96 /$ $[1,057 / 5,115,679])=8.44$. Second, we estimated the marginal standardized risks among fresh fish consumers and non-consumers in adolescence by predicting the risk for each subject in the control group and setting that person as exposed and unexposed and using his or her own confounder values and then averaging the predicted risks. Third, we estimated the marginal standardized OR using the standardized risks among fresh fish consumers and non-consum- 
ers in adolescence. The same steps in standardization were repeated for canned fish consumption as the second main exposure of interest. The validity of this estimate relies on the assumptions of no unmeasured confounding, and correct outcome model specification. We used the non-parametric bootstrap technique with 1,000 replications to derive $95 \% \mathrm{CI}$ via the 2.5 th and 97.5 th percentiles of the observed distribution of estimated standardized ORs.

\section{Model Specification and Covariate Balance Checking}

The locally weighted scatter plot smoothing algorithm and fractional polynomials were employed to detect any nonlinear association between continuous predictors and response in the PS and standardization outcome models. To assess covariate balance between the exposed and unexposed groups in the inverse-probability-of-treatment weighted sample, standardized mean difference,

$$
\mathrm{D}=\frac{100 \times\left|\overline{\mathrm{X}}_{\mathrm{e}}-\overline{\mathrm{X}}_{\mathrm{u}}\right|}{\sqrt{\frac{\mathrm{S}_{\mathrm{e}}^{2}+\mathrm{S}_{\mathrm{u}}^{2}}{2}}}
$$

and standardized prevalence difference,

$$
\mathrm{D}=\frac{100 \times\left|\widehat{\mathrm{P}_{\mathrm{e}}}-\widehat{\mathrm{P}_{\mathrm{u}}}\right|}{\sqrt{\frac{\widehat{\mathrm{P}_{e}}\left(1-\widehat{\mathrm{P}_{\mathrm{e}}}\right)+\widehat{\mathrm{P}_{\mathrm{u}}}\left(1-\widehat{\mathrm{P}_{\mathrm{u}}}\right)}{2}}}
$$

were calculated. It has been suggested that standardized differences $>10$ are not acceptable [35].

\section{Results}

During the 1.5-year study period, we identified 570 newly diagnosed MS patients. Of these, 547 (96\%) accepted to participate in our case-control study. In the end of the RDD process, 2,856 (64.3\%) of 4,457 random generated digit sequences were not active telephone lines. Of the 1,601 active ones, 91 (5.7\%) had no eligible person within their household, leaving 1,510 (94.3\%) contactable households with an eligible person from which 1,057 individuals participated in the study. Overall, this yields a response rate of $70 \%$ of eligible random digit numbers that have been included as study controls and have completed the main study checklist. While 195 (36.4\%) of cases reported fresh fish consumption in adolescence for $\geq 0.5$ serving size/week, 432 (45.9\%) of controls reported a similar experience. The reported adolescent SES was a little lower in cases than controls. While the proportion of passive smokers in adolescence was higher in cases, the proportion of participants who smoked in adolescence was not significantly different between the case and control groups (Table 1).
Table 2 shows the standardized differences of potential confounders between exposure after IPT weighting using controls and weighted total sample.

Before applying IPTW, the range of standardized differences of potential confounders was between 4.7 and 34.0. However, after IPT weighting using controls and the weighted total sample, the largest absolute standardized difference was only 1.0 and none of the corresponding $p$ values is significant (data not shown). Both weighting schemes have improved the balance of measured confounder dramatically. A very similar balance pattern was observed for confounders between levels of canned fish consumption in adolescence as the other study main exposure (data not shown).

The marginal effects of fresh and canned fish on MS using PS methods and MBS are presented in Table 3. The mean (SD) and range of weights for fresh fish were 1.01 $(0.24)$ and $(0.55-2.14)$ for both weighted case-control and control methods. Similarly, the mean (SD) and range of weights for canned fish were $1.00(0.26)$ and (0.54-2.92) for both weighted case-control and control methods (Table 3). Entering family history of MS or reported autoimmune disease to the final PS models had no impact on the distribution of stabilized weights, nor to the estimated marginal ORs and their corresponding $p$ values. These variables were therefore excluded from the DAG and the final models.

\section{Discussion}

In IPTW analysis, using both control and weighted case control methods, fresh fish consumption for $\geq 0.5$ serving size/week during adolescents reduced the risk of MS by $0.28 \%$. Similarly, consumption of more than one serving size tuna cans of any fish per month decreased the risk of MS by $0.26 \%$. Moreover, in the MBS method, fresh and canned fish consumptions during adolescents decreased the risk of MS by 29 and $28 \%$, respectively. There is no any information concerning the relevant induction time for fresh or canned fish intake on MS. However, when we reanalyzed in cases and controls older than 20 years, we have not seen any substantial change in the results. This could simply confirm the acknowledged protective role of fresh and canned fish intake during adolescence on MS development even for people aged more than 20 years old. In fact, all of the causal analytic approaches applied in this study have supported the protective role of fresh and canned fish consumptions in adolescence on MS onset with very similar effect size estimates. Fish is rich in 
Table 1. Characteristics of 547 MS cases and 1,057 general population controls, Tehran, 2013-2015

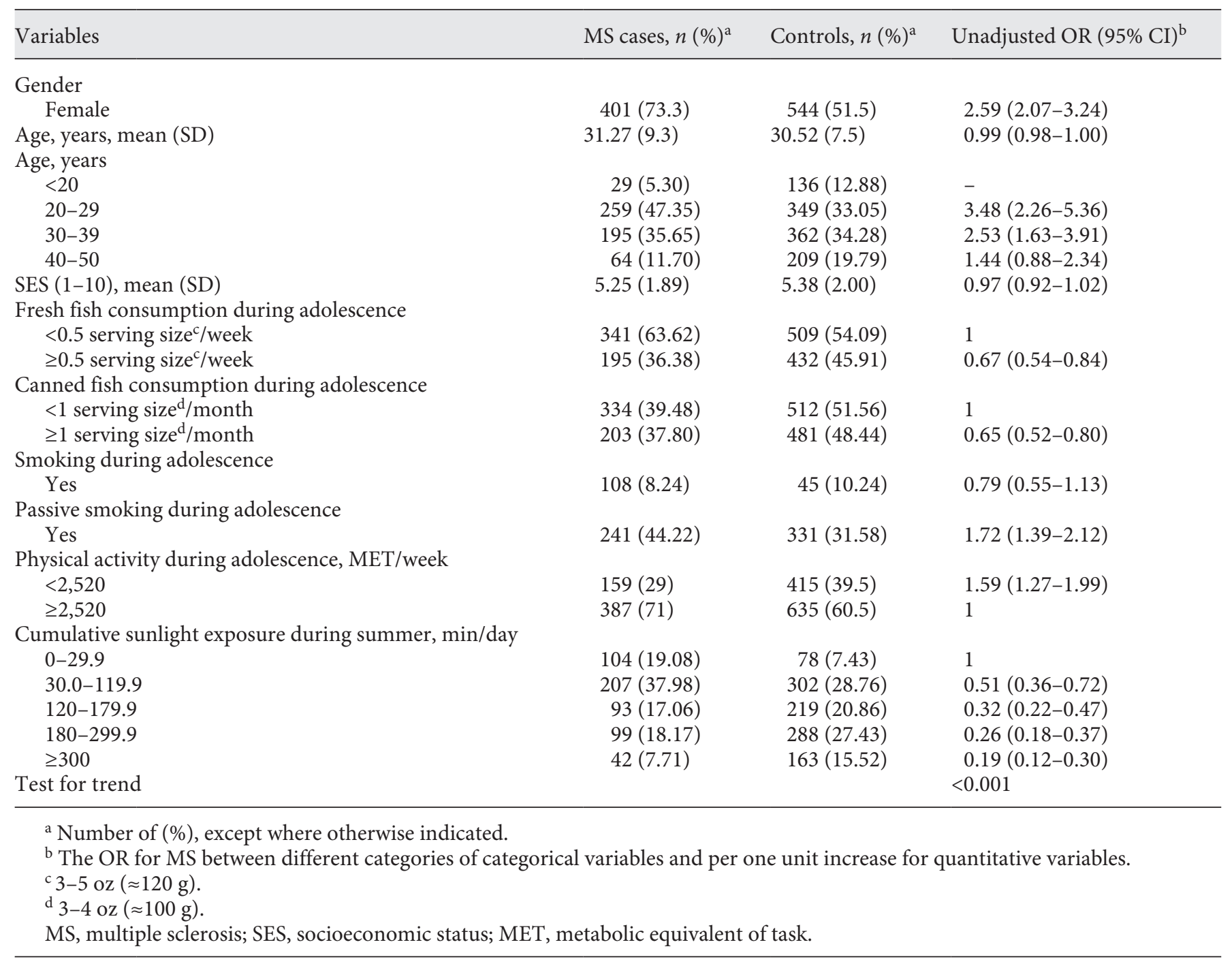

omega-3 fatty acids, and this important anti-inflammatory substance may decrease the risk of developing MS [36]. Similarly, the low level of vitamin D may be alleviated by fish consumption leading to a reduced risk of MS.

Although application of PS, as a prospective balancing score, in observational studies had been primarily restricted to cohort studies, its estimation and application in case-control studies has been discussed [12]. In fact it is possible to consistently estimate PS in case-control studies using (i) controls that are representative of the population that gave rise to the cases, and (ii) the total sample with inverse-probability weighting where weights are the inverse of case and control SF [12]. While, valid PS estimation in method (ii) requires knowledge of the sampling fraction of the study groups, PS estimation in method (i) is approximately correct under the rare disease assumption. Similarly, while MBS, also known as the parametric g-formula, has been introduced in cohort studies [12], it can be easily generalized to case-control studies if case and control SF are known [9]. The validity of the reported causal OR using IPW and MBS depends on the underlying assumptions including no residual confounding and correct model specification. The validity of IP weighted ORs also requires positivity. The IP weighted and model-based standardized OR estimates were very similar. This similarity does not guarantee the absence of model misspecification, but is reassuring, because it is unlikely that exposure and outcome models result in bias of the similar magnitude and direction. We included known risk factors associated with fresh and 
Table 2. IPTW standardized difference of covariates after weighting of sample, Tehran, 2013-2015

\begin{tabular}{|c|c|c|c|}
\hline \multirow[t]{3}{*}{ Characteristics } & \multicolumn{3}{|c|}{ Standardized difference } \\
\hline & \multirow[t]{2}{*}{ before IPTW } & \multicolumn{2}{|l|}{ after IPTW ${ }^{\mathrm{a}}$} \\
\hline & & $\begin{array}{l}\text { weighted cases } \\
\text { control method }\end{array}$ & control method \\
\hline Gender & 10.0 & 4.0 & 0.4 \\
\hline $\mathrm{Age}^{\mathrm{b}}$ & 10.9 & 5.0 & 0.2 \\
\hline SES in adolescence & 34.0 & 3.1 & 0.6 \\
\hline Sunlight exposure in adolescence & 8.9 & 8.1 & 0.1 \\
\hline Physical activity in adolescence & 10.3 & 1.4 & 1.4 \\
\hline Smoking in adolescence, yes/no & 4.7 & 2.5 & 0.4 \\
\hline Passive smoking in adolescence, yes/no & 6.4 & 6.1 & 0.7 \\
\hline
\end{tabular}

Table 3. Causal analysis of the effect of fresh and canned fish on MS using PS method and model-based standardization

\begin{tabular}{|c|c|c|c|c|c|c|}
\hline \multirow[t]{2}{*}{ Method } & \multicolumn{2}{|c|}{ Stabilized weights, mean (SD) } & \multicolumn{2}{|c|}{ Marginal OR (95\% CI) } & \multicolumn{2}{|l|}{$p$ value } \\
\hline & fresh fish & canned fish & fresh fish & canned fish & fresh fish & canned fish \\
\hline PS (control method) & $1.01(0.24)$ & $1.00(0.26)$ & $0.72(0.58-0.90)$ & $0.75(0.58-0.91)$ & 0.005 & 0.014 \\
\hline PS (weighted case control method) & $1.01(0.24)$ & $1.01(0.35)$ & $0.72(0.58-0.90)$ & $0.75(0.58-0.91)$ & 0.005 & 0.014 \\
\hline MBS & & & $0.72(0.58-0.91)$ & $0.73(0.59-0.94)$ & 0.008 & 0.006 \\
\hline
\end{tabular}

PS, propensity score; MBS, model-based standardization; MS, multiple sclerosis.

canned fish consumptions in adolescence in study controls in our exposure and outcome models. To avoid inefficiency and non-positivity problems, we did not select variables that were only associated with fresh and canned fish consumptions in adolescence in study controls but were not risk factors for MS [37]. The mean of IP stabilized weights based on both control and weighted casecontrol method were exactly 1 , a necessary condition for correct PS model specification. The similarity of the mean (SD) of the weights using control and weighted casescontrol method is unsurprising given the rarity of MS. Furthermore, the maximum of generated IPT weights in both methods for both fresh and canned fish was 2.36, which are less than $2 \%$ of the study sample size. There was no covariate with standardized differences more than $10 \%$ after IP weighting in our selected confounders, reinforcing the ignorable effect of confounding issue at least regarding measured confounders.

Our results should be interpreted with some limitations in mind. With all causal analyses, including those used in this study, it is only expected to remove the effect of measured, but not unknown or unmeasured confounders. Therefore, unmeasured and unknown covariates can still be imbalanced between groups defined based on the amount of fresh and canned fish consumptions leading to residual confounding. Moreover, the possibility of measurement error in the main study exposure as well as for other covariates due to recall bias should not be ignored. This limitation in obtaining dietary information during adolescence might be highlighted. Potential exposure assessment error (recall bias) might threat the validity of estimated ORs [38]. The differential or non-differential nature of the resulting misclassification is important in predicting the direction of the imposed bias as well as estimating the magnitude of the true ORs. If the recall error was random and independent from other covariates, the bias would be towards null. In that case, the reported finding would be underestimated, that is, the true ORs would be smaller than those reported in our results. However, differential recall bias could occur only if the respondents 
had some idea that fish could be a risk factor and so remembered this more often only if they were cases, which seems implausible. Nimptsch et al. [38] utilized the same method as we did for measuring meat consumption during participants' high school period. Although the validity of food frequency questionnaire in the case of adolescent diet has been approved in the US Nurses' Health Study II cohort [39], there are still some controversies regarding its validity [40]. Considering the imbalanced distribution of age categories between study groups, it is possible that some degree of recall bias still remained, due to the extended time that passed between the exposure period and the outcome assessment. Moreover, reporting bias as well as measurement error in self-reported covariates including drug abuse and alcohol intake can lead to important residual confounding. The direction of the bias due to measurement errors in exposure and confounders is unpredictable without knowledge of error structures. The possibility of selection bias is another issue threatening the validity of the findings of this study. RDD can access only a subset with residential phone lines, which implies a stable residence and a certain income level, perhaps leading to an imposed selection bias. However, the sampling fraction for cases was close to 1 with a response rate of $96 \%$. This could alleviate the possibility of selection bias at least in the case group. On the contrary, using RDD for selecting population-based controls can alleviate the possibility of selection bias. Although, the response rate in control group was significantly smaller than cases, compared with other studies, this may be considered as a satisfactory response rate [41]. As another limitation, we have used case and control SF in both IPTW and MBS even though we selected one control from each household using Kish method. This does not seem to introduce a substantial bias, as household size is not strongly correlated with either fish consumption or
MS conditional on the confounders used in the models including SES. Finally, it is less likely that the rather flexible modeling and the ascertainment of cases could be an important source for bias.

In conclusion, based on the results of PS and MBS analyses, fresh fish consumption in adolescence at the level of $\geq 0.5$ serving size per week decreased the incidence odds of MS by 0.72 times compared to those with less than this level of consumption. Similarly, consumption of one serving size canned tuna fish per month decreased the incidence odds of MS by 0.75 times compared to those with less than this level of consumption. However, considering the inherent limitations of case-control studies, the causal analysis using PS and MBS methods does not necessarily establish a causal role of the fresh and canned fish consumptions in the MS etiology. This finding reinforces the need for a public health educational program aimed at changing the nutritional habits of the general population and especially of young adults.

\section{Acknowledgments}

The authors appreciate the cooperation of the staff members of the IMSS.

\section{Disclosure Statement}

None declared.

\section{Funding Sources}

This work was supported by Tehran University of Medical Sciences and Multiple Sclerosis Research Center (MSRC; under grant number 93-04-10-240).

\section{References}

1 Browne P, Chandraratna D, Angood C, Tremlett H, Baker C, Taylor BV, Thompson AJ: Atlas of multiple Sclerosis 2013: a growing global problem with widespread inequity. Neurology 2014;83:1022-1024.

-2 Elhami SR, Mohammad K, Sahraian MA, Eftekhar H: A 20-year incidence trend (19892008) and point prevalence (March 20, 2009) of multiple sclerosis in Tehran, Iran: a population-based study. Neuroepidemiology 2011; 36:141-147.

3 Etemadifar M, Maghzi AH: Sharp increase in the incidence and prevalence of multiple scle- rosis in Isfahan, Iran. Mult Scler 2011;17: 1022-1027.

-4 Abdollahpour I, Nedjat S, Sahraian MA, Mansournia MA, Otahal P, van der Mei I: Waterpipe smoking associated with multiple sclerosis: a population-based incident case-control study. Mult Scler: 2017;23:1328-1335.

5 Hedström AK, Bäärnhielm M, Olsson T, Alfredsson L: Exposure to environmental tobacco smoke is associated with increased risk for multiple sclerosis. Mult Scler 2011;17:788-793.

6 Handel AE, Williamson AJ, Disanto G, Dobson R, Giovannoni G, Ramagopalan SV:
Smoking and multiple sclerosis: an updated meta-analysis. PLoS One 2011;6:e16149.

>7 Munger KL, Zhang SM, O'Reilly E, Hernan MA, Olek MJ, Willett WC, Ascherio A: Vitamin $\mathrm{D}$ intake and incidence of multiple sclerosis. Neurology 2004;62:60-65.

-8 Mansournia MA, Danaei G, Forouzanfar MH, Mahmoodi M, Jamali M, Mansournia N, Mohammad K: Effect of physical activity on functional performance and knee pain in patients with osteoarthritis: analysis with marginal structural models. Epidemiology 2012; 23:631-640.
The Effect of Fish Intake on Multiple Sclerosis
Neuroepidemiology 2018;50:111-118 DOI: $10.1159 / 000487640$ 
9 Rothman KJ, Greenland S, Lash TL: Modern Epidemiology. Philadelphia, Lippincott Williams \& Wilkins, 2008.

-10 Rose S, van der Laan M: A double robust approach to causal effects in case-control studies. Am J Epidemiol 2014;179:663-669.

11 Newman SC: Causal analysis of case-control data. Epidemiol Perspect Innovs 2006;27:2.

12 Mannsson R, Joffe MM, Sun W, Hennessy S: On the estimation and use of propensity scores in case-control and case-cohort studies. Am J Epidemiol 2007;166:332-339.

-13 Mansournia MA, Etminan M, Danaei G, Kaufman JS, Collins G: Handling time varying confounding in observational research. BMJ 2017;359:j4587.

14 Gharibzadeh S, Mohammad K, Rahimiforoushani A, Amouzegar A, Mansournia MA: Standardization as a tool for causal inference in medical research. Arch Iran Med. 2016;19: 666-670.

-15 Heydarpour P, Mohammad K, Yekaninejad MS, Elhami SR, Khoshkish S, Sahraian MA: Multiple sclerosis in Tehran, Iran: a joinpoint trend analysis. Mult Scler 2013;20:512.

-16 Polman CH, Reingold SC, Banwell B, Clanet M, Cohen JA, Filippi M, Fujihara K, Havrdova E, Hutchinson M, Kappos L: Diagnostic criteria for multiple sclerosis: 2010 revisions to the McDonald criteria. Ann Neurol 2011; 69:292-302

17 Wacholder S, Silverman DT, McLaughlin JK, Mandel JS: Selection of controls in case-control studies. II. Types of controls. Am J Epidemiol 1992;135:1029-1041.

18 Clagett B, Nathanson KL, Ciosek SL, McDermoth M, Vaughn DJ, Mitra N, Weiss A, Martonik R, Kanetsky PA: Comparison of address-based sampling and random-digit dialing methods for recruiting young men as controls in a case-control study of testicular cancer susceptibility. Am J Epidemiol 2013; 178:1638-1647.

19 Gundersen DA, ZuWallack RS, Dayton J, Echeverría SE, Delnevo CD: Assessing the feasibility and sample quality of a national randomdigit dialing cellular phone survey of young adults. Am J Epidemiol 2014;179:39-47.

20 Kish L: A procedure for objective respondent selection within the household. Journal of the American Statistical Association 1949;44: 380-387.
21 Binson D, Canchola JA, Catania JA: Random selection in a national telephone survey: a comparison of the Kish, next-birthday, and last-birthday methods. J Official Stat Stockh 2000;16:53-60.

22 Pugliatti M, Casetta I, Drulovic J, Granieri E, Holmøy T, Kampman MT, Landtblom AM, Lauer K, Myhr KM, Parpinel M, Pekmezovic T, Riise T, Zhu B, Wolfson C: A questionnaire for multinational case-control studies of environmental risk factors in multiple sclerosis (EnvIMS-Q). Acta Neurol Scand Suppl 2012; 195:43-50.

23 Feskanich D, Rimm EB, Giovannucci EL, Colditz GA, Stampfer MJ, Litin LB, Willett WC: Reproducibility and validity of food intake measurements from a semiquantitative food frequency questionnaire. J Am Diet Assoc 1993;93:790-796.

24 Greenland S, Pearl J, Robins JM: Causal diagrams for epidemiologic research. Epidemiology 1999;10:37-48.

25 Booth ML, Ainsworth BE, Pratt M, Ekelund U, Yngve A, Sallis JF, Oja P: International physical activity questionnaire: 12-country reliability and validity. Med Sci Sports Exerc 2003; 195:3508-1381.

26 Ainsworth BE, Haskell WL, Whitt MC, Irwin ML, Swartz AM, Strath SJ, O'Brien WL, Bassett DR, Schmitz KH, Emplaincourt PO: Compendium of physical activities: an update of activity codes and MET intensities. Med Sci Sports Exerc 2000;32(suppl 9):S498 S504.

-27 Strong WB, Malina RM, Blimkie CJ, Daniels SR, Dishman RK, Gutin B, Hergenroeder AC, Must A, Nixon PA, Pivarnik JM, Rowland T, Trost S, Trudeau F: Evidence based physical activity for school-age youth. J Pediatr 2005; 146:732-737.

28 Adler NE, Epel ES, Castellazzo G, Ickovics JR: Relationship of subjective and objective social status with psychological and physiological functioning: preliminary data in healthy white women. Health psychol 2000;19:586592.

29 Mansournia MA, Altman DG: Inverse probability weighting. BMJ 2016;352:i189.

30 Månsson R, Joffe MM, Sun W, Hennessy S: On the estimation and use of propensity scores in case-control and case-cohort studies. Am J Epidemiol 2007;166:332-339.
31 Elhami SR, Mohammad K, Sahraian MA, Eftekhar H: A 20-year incidence trend (19892008) and point prevalence (March 20, 2009) of multiple sclerosis in Tehran, Iran: a population-based study. Neuroepidemiology 2010; 36:141-147.

32 Rubin DB: Estimating causal effects from large data sets using propensity scores. Ann Intern Med 1997;127:757-763.

33 Rubin DB, Thomas N: Matching using estimated propensity scores: relating theory to practice. Biometrics 1996;52:249-264.

34 Robins J: A new approach to causal inference in mortality studies with a sustained exposure period - application to control of the healthy worker survivor effect. Mathematical Modelling 1986; 7:1393-1512.

35 Austin PC: The relative ability of different propensity score methods to balance measured covariates between treated and untreated subjects in observational studies. Med Decis Making 2009;29:661-677.

36 Calder PC: n-3 polyunsaturated fatty acids, inflammation, and inflammatory diseases. Am J Clin Nutr 2006;83(suppl 6):1505S1519S.

37 Brookhart MA, Schneeweiss S, Rothman KJ, Glynn RJ, Avorn J, Stürmer T: Variable selection for propensity score models. Am J Epidemiol 2006;163:1149-1156.

38 Nimptsch K, Bernstein AM, Giovannucci E, Fuchs CS, Willett WC, Wu K: Dietary intakes of red meat, poultry, and fish during high school and risk of colorectal adenomas in women. Am J Epidemiol 2013;178:172183.

39 Maruti SS, Feskanich D, Colditz GA, Frazier AL, Sampson LA, Michels KB, Hunter DJ, Spiegelman D, Willett WC: Adult recall of adolescent diet: reproducibility and comparison with maternal reporting. Am J Epidemiol 2005;161:89-97.

40 Chavarro JE, Michels KB, Isaq S, Rosner BA, Sampson L, Willey C, Tocco P, Willett WC, Chumlea WC: Validity of maternal recall of preschool diet after 43 years. Am J Epidemiol 2009;169:1148-1157.

41 Massey JT, O’Connor D, Krotki K: Response Rates in Random Digit Dialing (RDD) Telephone Surveys: Proceedings of the American Statistical Association, Section on Survey Research Methods, 1997, pp 707-712. 